\title{
Reactions of $\mathrm{MoO}_{2} \mathrm{Cl}_{2}$ and $\mathrm{MoOCl}_{4}$ with 2-Mercaptopyridine, 4-Phenylimidazole-2-thiol and 6-Mercaptopurine monohydrate
}

\author{
DEEPIKA RANI ${ }^{1}$, GURSHARAN SINGH ${ }^{2 *}$ and SEEMA SHARMA ${ }^{3}$
}

\author{
${ }^{1}$ Research Scholar Registered with Punjab Technical University, Kapurthala, India. \\ ${ }^{2}$ Former Dean Academic Affairs, Maharaja Ranjit Singh Punjab Technical University, Bathinda, India. \\ ${ }^{3}$ Department of Applied Chemistry, Giani Zail Singh Campus College of Engineering \\ \& Technology, Dabwali Road, MRSPTU Bathinda-151001, India. \\ ${ }^{*}$ Corresponding author E-mails: gursharans82@ gmail.com \\ http://dx.doi.org/10.13005/ojc/370105
}

(Received: January 18, 2021; Accepted: February 19, 2021)

\begin{abstract}
$\mathrm{MoO}_{2} \mathrm{Cl}_{2} / \mathrm{MoOCl}_{4}$ have been reacted with 4-phenylimidazole-2-thiol/6-mercaptopurine monohydrate/2-mercaptopyridine in acetonitrile solvent in unimolar/bimolar proportions at room temperature. The products thus obtained are: $\mathrm{MoOCl}_{3}\left(\mathrm{C}_{9} \mathrm{H}_{8} \mathrm{~N}_{2} \mathrm{~S}\right)$, [1]; $\mathrm{Mo}_{2} \mathrm{O}_{3} \mathrm{Cl}_{6}\left(\mathrm{C}_{9} \mathrm{H}_{7} \mathrm{~N}_{2} \mathrm{~S}\right)$ $\left(\mathrm{CH}_{3} \mathrm{CN}\right)_{2}$,[2]; $\mathrm{Mo}_{2} \mathrm{O}_{3} \mathrm{Cl}_{8}\left(\mathrm{C}_{9} \mathrm{H}_{7} \mathrm{~N}_{2} \mathrm{~S}\right)_{2}\left(\mathrm{CH}_{3} \mathrm{CN}\right)_{2}$, [3] and $\mathrm{Mo}_{2} \mathrm{O}_{4} \mathrm{Cl}_{4}\left(\mathrm{C}_{5} \mathrm{H}_{4} \mathrm{NS}-\mathrm{SN}_{4} \mathrm{C}_{5}\right)$, [4]. These products were studied by various techniques: infrared, proton NMR, liquid/gas chromatographymass spectrometry, elemental analyses. Owing to the sensitivity of the products to air and moisture, the reactions and work ups were performed in vacuum line purged with oxygen by flushing dry nitrogen in it. lons observed in mass spectrum are concurrent with the depicted formulae.
\end{abstract}
Keywords: $\mathrm{MoO}_{2} \mathrm{Cl}_{2}, \mathrm{MoOCl}_{4}$, 2-mercaptopyridine, 4-phenylimidazole-2-thiol, 6-mercaptopurine monohydrate, Acetonitrile solvent, Infrared, proton NMR, DMSO-d, Liquid/gas chromatography-mass spectrometry.

\section{INTRODUCTION}

6-Mercaptopurine ring system may be considered as if a pyrimidine ring has been fused to an imidazole ring. Electrons of 6-mercaptopurine are highly delocalized. The ring is susceptible to both electrophilic and nucleophilic attacks. 6-Mercaptopurine ${ }^{1-2}$ is used as chemotherapy drugs for treatment of autoimmune diseases and cancer like leukemia, ulcerative colitis and
Crohn's disease. Mercaptopurine is sold as purinethol. It is a class of medication known as purine antagonists and works by stopping the growth of cancer cells. Many transition metal complexes of 6-mercaptopurine are reported ${ }^{3-4}$. Some of transition metal complexes of 6-mercaptopurine have higher anticancer activity than that of 6-mercaptopurine ${ }^{5-8}$. Divalent transition metals coordinate ${ }^{5,7,9}$ through $\mathrm{S}$ and $\mathrm{N}$ atoms of 6-mercaptopurine.

This is an Open Access article licensed under a Creative Commons license: Attribution 4.0 International (CC- BY). Published by Oriental Scientific Publishing Company @ 2018

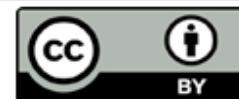


<smiles>S=c1nc[nH]c2nc[nH]c12</smiles>

Heterocyclic thioamides like 4-pheny -limidazole-2-thiol having $\mathrm{N}$ and S-donor ligands are biologically active and are used as anti-thyroidal agents ${ }^{10}$. Imidazothiazole structural unit containing heterocyclic compounds are biologically active ${ }^{11}$. Many enzymes and receptors ${ }^{12-15}$ can be inhibited by them. They are used in diuretic ${ }^{16}$, fungicidal ${ }^{17}$, antihelmintic ${ }^{18}$, antitumor ${ }^{19-24}$, antidiabetic ${ }^{25}$ and antimicrobial ${ }^{26-27}$ drugs.

There are many biochemical applications ${ }^{28-30}$ of metal complexes with thioligands.

\section{AIM of investigation}

$\mathrm{MoO}_{2} \mathrm{Cl}_{2}$ and $\mathrm{MoOCl}_{4}$ are known to react with a variety of ligands. The author earlier investigated ${ }^{31-37}$ reactions of $\mathrm{MoO}_{2} \mathrm{Cl}_{2}$ with various diaminoalkanes, alkanediols, amides, imides, thiols and aromatic azoles.

The author earlier also investigated ${ }^{31-33,38-39}$ reactions of $\mathrm{MoOCl}_{4}$ with various diaminoalkanes, amides, imides, alkylpyridines, mercaptopyridine, mercaptopyridine-N-oxide sodium, 2-thiazoline-2thiol, alkylpyrrolidine, alkylpiperidine and aromatic azoles.

The author has reported earlier also molybdenum compounds containing 4-pheny -limidazole-2-thiol, 6-mercaptopurine monohydrate and 2-mercaptopyridine.

In view of the wide applications of the transition metal complexes, now author has prepared molybdenum complexes of 2-Mercaptopyridine, 4-Phenylimidazole-2-thiol and 6-Mercaptopurine monohydrate on reaction with $\mathrm{MoO}_{2} \mathrm{Cl}_{2}$ and $\mathrm{MoOCl}_{4}$. The complexes have been characterised by elemental analysis, Mass, IR and NMR techniques. All preparations and work ups have been done under rigorous moisture/air free environment.

\section{MATERIALS AND METHODS}

$\mathrm{MoO}_{2} \mathrm{Cl}_{2}, \mathrm{MoOCl}_{4}$, 4-phenylimidazole2-thiol, 6-mercaptopurine monohydrate and 2-mercaptopyridine used were manufactured by Sigma-Aldrich. We used them without any further treatment. Owing to the sensitivity of the products to air and moisture, the reactions and work ups were performed in vacuum line purged with oxygen by flushing dry nitrogen in it. The reactions were carried out for 6-8 $\mathrm{h}$ with continuous stirring using pressure stabilised dropping funnel. The products were filtered through filtration unit fitted with G-4 crucible and isolated.

Molybdenum was determined by oxinate ${ }^{40}$ gravimetric method. Chlorine was determined by silver chloride ${ }^{40}$ gravimetric method. Thermo Finnigan Elemental Analyser was used to determine other elements. Perkin-Elmer 400 FTIR Spectrometer, in the range $4000-400 \mathrm{~cm}^{-1}$ was used to obtain spectra using $\mathrm{KBr}$ disks. ${ }^{1} \mathrm{H}-\mathrm{NMR}$ spectra were recorded in solvent DMSO- $\mathrm{d}_{6}$ using Brucker Avance-II 400 NMR. Liquid Chromatography-Mass spectra were obtained in the range $0-1100 \mathrm{~m} / \mathrm{z}$. These facilities were provided by Panjab University, Chandigarh (India).

\section{Preparation of compounds ${ }^{1-4}$}

Disproportionation/rearrangement might have occurred during the course of reactions. The source of the products is indicated below the products.

$$
\begin{aligned}
& \mathrm{M}_{00 C l}+\mathrm{C}_{9} \mathrm{H}_{8} \mathrm{~N}_{2} \mathrm{~S} \underset{1: \mathrm{C}}{\stackrel{\mathrm{CH}_{3} \mathrm{CN}}{\longrightarrow}} \mathrm{M} 00 C l_{3}\left(\mathrm{C}_{9} \mathrm{H}_{8} \mathrm{~N} \mathrm{~N}_{2} \mathrm{~S}\right),[1] \\
& \text { 4-Phenylimidazole-2-thiol Black } \\
& \mathrm{M} 0 O C l_{4}+\mathrm{C}_{5} \mathrm{H}_{4} \mathrm{~N}_{4} \mathrm{~S} \cdot \mathrm{H}_{2} \mathrm{O} \underset{1: 2}{\stackrel{\mathrm{CH}_{3} \mathrm{CN}}{\longrightarrow}} \mathrm{Mo}_{2} \mathrm{O}_{3} \mathrm{Cl}_{6}\left(\mathrm{C}_{5} \mathrm{H}_{2} \mathrm{~N}_{4} \mathrm{~S}\right)\left(\mathrm{CH}_{3} \mathrm{CN}\right)_{2},[2] \\
& \text { 6-Mercaptopurine monohydrate Blackish brown } \\
& \mathrm{MoOCl}_{4}+\mathrm{C}_{5} \mathrm{H}_{4} \mathrm{~N}_{4} \mathrm{~S}_{2} \mathrm{H}_{2} \mathrm{O} \stackrel{\mathrm{C}}{\stackrel{\mathrm{CH}}{3} \mathrm{CN}} \underset{\mathrm{l}}{\longrightarrow} \mathrm{M}_{2} \mathrm{O}_{3} \mathrm{Cl}_{8}\left(\mathrm{C}_{5} \mathrm{H}_{2} \mathrm{~N}_{4} \mathrm{~S}\right)_{2}\left(\mathrm{CH}_{3} \mathrm{CN}\right)_{2},[3] \\
& \text { 6-Mercaptopurine monohydrate Coffeebrown }
\end{aligned}
$$

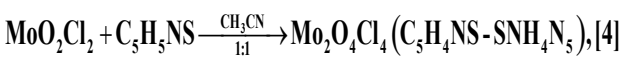

$$
\begin{aligned}
& \text { 2-Mercaptopyridine Black }
\end{aligned}
$$

\section{RESULTS AND DISCUSSIONS}

\section{Analytical Measurements}

Estimation of elements (percentage) are given in Table 1. Theoretical values are given in parenthesis. 
Table 1: (Elemental Analysis)

\begin{tabular}{|c|c|c|c|c|c|c|}
\hline Products & Mo & $\mathrm{Cl}$ & $\mathrm{C}$ & $\mathrm{H}$ & $\mathrm{N}$ & $S$ \\
\hline $\mathrm{MoOCl}_{3}\left(\mathrm{C}_{9} \mathrm{H}_{8} \mathrm{~N}_{2} \mathrm{~S}\right),[1]$ & 23.66 & 26.78 & 28.13 & 2.78 & 6.13 & 7.23 \\
\hline$($ Black/394.5) & -24.33 & -27 & -27.38 & -2.03 & -7.1 & -8.11 \\
\hline $\mathrm{Mo}_{2} \mathrm{O}_{3} \mathrm{Cl}_{6}\left(\mathrm{C}_{9} \mathrm{H}_{7} \mathrm{~N}_{2} \mathrm{~S}\right)\left(\mathrm{CH}_{3} \mathrm{CN}\right)_{2}$,[2] & 27.53 & 30.13 & 14.95 & 1.65 & 12.78 & 4.54 \\
\hline (Blackish brown/685.0) & -28.02 & -31.09 & -15.76 & -1.16 & -12.26 & -4.67 \\
\hline $\mathrm{Mo}_{2} \mathrm{O}_{3} \mathrm{Cl}_{8}\left(\mathrm{C}_{9} \mathrm{H}_{7} \mathrm{~N}_{2} \mathrm{~S}\right)_{2}\left(\mathrm{CH}_{3} \mathrm{CN}\right)_{2},[3]$ & 20.78 & 30.73 & 17.66 & 1.17 & 15.14 & 3.23 \\
\hline (Coffee brown/906.0) & -21.19 & -31.34 & -18.54 & -1.1 & -15.45 & -3.53 \\
\hline $\mathrm{Mo}_{2} \mathrm{O}_{4} \mathrm{Cl}_{4}\left(\mathrm{C}_{5} \mathrm{H}_{4} \mathrm{NS}-\mathrm{SN}_{4} \mathrm{C}_{5}\right),[4]$ & 31.67 & 22.6 & 18.54 & 1.48 & 4.34 & 9.55 \\
\hline$($ Black/618.0) & -31.06 & -22.97 & -19.41 & -1.29 & -4.53 & -10.35 \\
\hline
\end{tabular}

\section{FTIR Spectra}

Absorption at $3137 \mathrm{~cm}^{-1}$ in[1] refers to $\mathrm{N}-\mathrm{H}$ stretching of 4-phenylimidazole-2-thiol ${ }^{37,41-43}$ (Table 2). There is no $\mathrm{v}(\mathrm{S}-\mathrm{H})$ in the range $2551-2602 \mathrm{~cm}^{-1}$ of[1] indicating S-H group is not present it. Peaks at $1261 \mathrm{~cm}^{-1}$ and $1106 \mathrm{~cm}^{-1}$ in [1] correspond to $\mathrm{C}=\mathrm{S}$ stretching. $v(C=O)$ is higher than $v(C=S)$, because carbonyl bond is stronger and more polar than thiocarbonyl bond. Carbonyl bond absorptions are more intense than that of thiocarbonyl bond. C-S stretching is detected at $761 \mathrm{~cm}^{-1}$. C-S stretching observed points out to the existence of Mo-S bonds. $v(\text { Mo-S })^{9,49}$ appears at $498 \mathrm{~cm}^{-1}$. Terminal $\mathrm{Mo}=\mathrm{O}$ group absorbs ${ }^{44}$ in the span $991 \mathrm{~cm}^{-1}$ $-1008 \mathrm{~cm}^{-1}$. Mo=O stretching ${ }^{45-47}$ was observed at $986 \mathrm{~cm}^{-1}$. There is thiol-thione tautomerism in imidazole-2-thiones ${ }^{37,43}$. Mo $=0$ stretching shows downward shift owing to $\mathrm{S} \rightarrow$ Mo coordination 37,48 of 4-phenylimidazole-2-thiol. Ligand coordination is trans to $\mathrm{Mo}=\mathrm{O}$. It implies that 4-phenylimidazole-2thione reacts in thiol form. This fact is further evident by the higher value of $v(C=N)$.

Table 2: (FTIR frequencies in $\mathrm{cm}^{-1}$ )

\begin{tabular}{ccc}
\hline Mode & (4-Phenylimidazole-2-thiol) $^{37,41-43}$ & \\
\hline$v(\mathrm{~N}-\mathrm{H})$ & $3129,3248 \mathrm{~s}$ & $3137.28 \mathrm{v} \mathrm{s}$ \\
$\mathrm{v}(\mathrm{S}-\mathrm{H})$ & $1560,1502,1463$ & $1621.30 \mathrm{~s}, 1597.31 \mathrm{~s}$, \\
$\mathrm{v}(\mathrm{C}=\mathrm{N}), \mathrm{v}(\mathrm{C}=\mathrm{C})$ & 1261,1109 & $1505.38 \mathrm{~m}, 1457.37 \mathrm{~m}$ \\
& 780 & $1261 \mathrm{~m}, 1106 \mathrm{~m}$ \\
$\mathrm{v}(\mathrm{C}=\mathrm{S})$ & & $761.27 \mathrm{v} \mathrm{s}$ \\
$\mathrm{v}(\mathrm{C}-\mathrm{S})$ & & $498.44 \mathrm{v} \mathrm{w}$ \\
$\mathrm{v}(\mathrm{Mo}-\mathrm{S})^{9,49}$ & & $986.30 \mathrm{v} \mathrm{s}$ \\
\hline
\end{tabular}

Absorptions at $3400 \mathrm{~cm}^{-1}$ in [2] and 3407 $\mathrm{cm}^{-1}$ in [3] suggest $\mathrm{v}(\mathrm{N}-\mathrm{H})$ of pyrimidine ring of 6-mercaptopurine ${ }^{9,50,58} . v(\mathrm{~N}-\mathrm{H})$ of imidazole ring are missing due to absence of $\mathrm{N}-\mathrm{H}$ bond of imidazole ring (Table 3). This shows that there is Mo-N bond formation. No $v(\mathrm{~S}-\mathrm{H})$ peak has been observed in [2] and [3]. This means that 6-mercaptopurine has participated in thiol form. This is further supported by higher $\mathrm{C}=\mathrm{N}$ stretching in these complexes. Lower $\mathrm{N}-\mathrm{H}$ stretching implies coordination ${ }^{50}$ of 6-mercaptopurine to molybdenum. $v(\mathrm{Mo}-\mathrm{S})^{9,49}$ appears at $729 \mathrm{~cm}^{-1}$ and $728 \mathrm{~cm}^{-1}$ in [2] and [3], respectively. $\mathrm{v}(\mathrm{Mo}=\mathrm{O})$ absorptions at $973 \mathrm{~cm}^{-1}$ in [2] and $970 \mathrm{~cm}^{-1}$ [3], reveal terminal $(\mathrm{Mo}=\mathrm{O})^{45-47}$ in them.

Table 3: (FTIR frequencies in $\mathrm{cm}^{-1}$ )

\begin{tabular}{|c|c|c|c|}
\hline Mode & 6-Mercaptopurine monohydrate ${ }^{9,50,58}$ & [2] & [3] \\
\hline$v(\mathrm{~N}-\mathrm{H})$ Imidazole & 3523 & & \\
\hline $\mathrm{v}(\mathrm{N}-\mathrm{H})$ Pyrimidine & 3376 & 3400.5 v s & 3407.1 v s \\
\hline$v(C-H)$ & $3095.0,2993.8$ & & \\
\hline$v(\mathrm{~S}-\mathrm{H})$ & 2671.5 & & \\
\hline$v(C=C)$ & 1669.7 & & \\
\hline$v(\mathrm{C}=\mathrm{N})$ Imidazole & 1620 & 1626.8 v.s. & 1626.1 v.s. \\
\hline$v(C=N)$ Pyrimidine & 1393 & $1402.1 \mathrm{~m}$ & $1401.6 \mathrm{~m}$ \\
\hline$v(C-N)$ & 1343.8 & $1335.2 \mathrm{w}$ & $1334.9 \mathrm{w}$ \\
\hline $\mathrm{v}(\mathrm{N}-\mathrm{H})$ & 1526.6 & $1504.1 \mathrm{sh}$ & $1504.6 \mathrm{sh}$ \\
\hline$v(C=S)$ & 1193 & $1027.24 \mathrm{w}$ & $1027.1 \mathrm{w}$ \\
\hline$v(\text { Mo-S })^{9,49}$ & & $729.1 \mathrm{~m}$ & $728.6 \mathrm{~m}$ \\
\hline$v(\mathrm{Mo}-\mathrm{N})^{9}$ & & $496.2 \mathrm{w}$ & $496.9 \mathrm{w}$ \\
\hline Terminal $v(\mathrm{Mo}=\mathrm{O})^{45-47}$ & & 973.1 & $970.4 \mathrm{~s}$ \\
\hline
\end{tabular}


2-Mercaptopyridine ${ }^{33,51-55}$ shows $v(\mathrm{~N}-\mathrm{H})$ at $3177 \mathrm{~cm}^{-1}$ and $v(\mathrm{~S}-\mathrm{H})$ at $2708 \mathrm{~cm}^{-1}$ (Table 4). Bands at $3383 \mathrm{~cm}^{-1}$ shows that [4] has $\mathrm{N}-\mathrm{H}$ group. $v(\mathrm{~S}-\mathrm{H})$ around $2708 \mathrm{~cm}^{-1}$ is missing pointing to $\mathrm{S}-\mathrm{H}$ group absence in [4]. $\mathrm{v}(\mathrm{Mo}=\mathrm{O})$ peak at $983 \mathrm{~cm}^{-1}$ reveals presence of terminal $\mathrm{Mo}=\mathrm{O}$ group $^{45-47}$ [4].

Table 4: (FTIR frequencies in $\mathrm{cm}^{-1}$ )

\begin{tabular}{|c|c|c|}
\hline Mode & $(2-M e r c a p t o p y r i d i n e)^{33,51-55}$ & [4] \\
\hline$v(\mathrm{~N}-\mathrm{H})$ & 3177 & 3383 vs \\
\hline$v(\mathrm{C}-\mathrm{H})$ & $3053,2928,2880$ & $3128.2 \mathrm{~s}, 2889.1 \mathrm{~m}$ \\
\hline$v(S-H)$ & $2708 \mathrm{~m}$ & \\
\hline $\begin{array}{l}\text { Ring breathing modes \& Hydrogen in plane wagging } \\
v(C=N) \text { ring }\end{array}$ & $\begin{array}{c}1614 \mathrm{~s}, 1577 \mathrm{vs}, 1503 \mathrm{~s}, 1447 \mathrm{~s}, 1419 \mathrm{~s} \\
1274 \mathrm{~m}\end{array}$ & $\begin{array}{c}1604.1 \mathrm{~s}, 1578.2 \mathrm{~s}, 1496.2 \mathrm{~m} \\
1273.2 \mathrm{w}\end{array}$ \\
\hline$v(\mathrm{C}-\mathrm{H})$ in plane bending & $1247 \mathrm{~m}$ & $1227.4 \mathrm{w}$ \\
\hline$v(\mathrm{C}=\mathrm{S})$ & 1187 vs, 1144 vs & $1177.3 \mathrm{w}$ \\
\hline$v(C-S)$, Hydrogen out of plane bending out of plane & 746,614 & 767 vs, 707.3 w \\
\hline Terminal $v(\mathrm{Mo}=\mathrm{O})^{45-47}$ & & $983.2 \mathrm{~s}$ \\
\hline
\end{tabular}

\section{${ }^{1} \mathrm{H}$ NMR Spectra}

Chemical shift of 4-phenylimidazole-2thiol $^{37,56-57} \mathrm{~N}-\mathrm{H}$ occurs at $12.9 \mathrm{\delta}$. alcoholic, phenolic, amino and thiolic protons have no specific chemical shift, because these are labile and. Spectrum is generally recorded in a solvent, $\mathrm{N}-\mathrm{H}$ chemical shift (Table 5) is missing in [1]. S-H in 4-phenylimidazole-2thiol has chemical shift at $12.14 \mathrm{\delta}$. S-H peak is missing in [1]. S-H group does not exist in [1]. [1] shows upfield chemical shift of ring protons and $\mathrm{H}-5$.

Table 5: ('H NMR absorptions in ppm)

\begin{tabular}{ccc}
\hline Protons & (4-Phenylimidazole-2-thiol) $^{37,56-57}$ & {$[1]$} \\
\hline $\mathrm{N}-\mathrm{H}$ & 12.99 & \\
$\mathrm{~S}-\mathrm{H}$ & 12.14 & \\
$\mathrm{H}-5$ & 7.51 & 7.39 \\
Aromatic H-2 and H-6 & 8.13 & 7.72 \\
Aromatic H-3 and H-5 & 7.51 & 7.39 \\
Aromatic H-4 & 7.41 & \\
\hline
\end{tabular}

6-Mercaptopurine monohydrate ${ }^{58}$ aromatic $\mathrm{N}-\mathrm{H}$ absorption shits to $7.8 \delta, 7.15 \delta$ in [2] and [3], respectively showing that this $\mathrm{N}-\mathrm{H}$ is not involved in bonding (Table 6). S-H peak is missing in [2] and [3], indicating presence of Mo-S bond in them.

Table 6: ( ${ }^{1} \mathrm{H}$ NMR absorptions in ppm)

\begin{tabular}{cccc}
\hline Protons & $\begin{array}{c}\text { 6-Mercaptopurine } \\
\text { monohydrate }\end{array}$ & [2] & {$[3]$} \\
\hline $\mathrm{N}-\mathrm{H}$ (Arom) & 7.2 & 7.15 & 7.92 \\
$\mathrm{~S}-\mathrm{H}$ & 1.2 & & \\
$\mathrm{~N}-\mathrm{H}$ & 1.4 & 1.85 & 1.84 \\
$\mathrm{CH}_{3} \mathrm{CN}$ & & 2.05 & 2.01 \\
\hline
\end{tabular}

Comparison of 2-mercaptopyridine ${ }^{33,53}$ spectrum with that of [4] shows that absorptions move downfield (Table 7). There is no $\mathrm{N}-\mathrm{H}$ peak. Deshielding is due to an increase in $\pi$ electron density on coordination in the $\mathrm{C}-\mathrm{N}$ bond.

\begin{tabular}{lcr}
\multicolumn{3}{c}{ Table 7: ('H NMR absorptions in ppm) } \\
\hline Protons & (2-Mercaptopyridine) $^{3,53}$ & {$[4]$} \\
\hline $\mathrm{N}-\mathrm{H}$ & & \\
$\mathrm{C}_{3}-\mathrm{H}$ & 7.32 & 7.67 \\
$\mathrm{C}_{4}-\mathrm{H}$ & 6.81 & 7.28 \\
$\mathrm{C}_{5}-\mathrm{H}$ & 7.47 & 7.81 \\
$\mathrm{NC}-\mathrm{H}$ & 7.69 & 8.48 \\
\hline
\end{tabular}

Table 8: (Fragmentation)

\begin{tabular}{|c|c|}
\hline Comp. & \\
\hline$[1]$ & $\begin{array}{l}{\left[\mathrm{MoOCl}_{3}\left(\mathrm{C}_{9} \mathrm{H}_{7} \mathrm{~N}_{2} \mathrm{~S}\right)\right] \rightarrow\left[\mathrm{SOCl}_{2}\right]^{+} \rightarrow\left[\mathrm{SOCl}^{+}\right.} \\
{[1](\mathrm{F} \mathrm{.W}=394.5) \quad \mathrm{m} / \mathrm{z}=118.9 \mathrm{~m} / \mathrm{z}=84.0}\end{array}$ \\
\hline \multirow[t]{6}{*}[2]{} & $\begin{array}{c}{\left[\mathrm{Mo}_{2} \mathrm{O}_{3} \mathrm{Cl}_{6}\left(\mathrm{C}_{5} \mathrm{H}_{2} \mathrm{~N}_{4} \mathrm{~S}\right)\left(\mathrm{CH}_{3} \mathrm{CN}\right)_{2}\right]^{+} \stackrel{-2 \mathrm{Cl}}{\longrightarrow}\left[\mathrm{Mo}_{2} \mathrm{O}_{3} \mathrm{Cl}_{4}\left(\mathrm{C}_{5} \mathrm{H}_{2} \mathrm{~N}_{4} \mathrm{~S}\right)\left(\mathrm{CH}_{3} \mathrm{CN}\right)_{2}\right]^{+}} \\
\mathrm{m} / \mathrm{z}=610.19 \\
{[2](\mathrm{F} . W .=684.21)} \\
\downarrow\end{array}$ \\
\hline & {$\left[\mathrm{C}_{5} \mathrm{H}_{4} \mathrm{~N}_{4} \mathrm{~S}\right]^{+}+\left[\mathrm{Mo}_{2} \mathrm{OCl}_{4}\left(\mathrm{CH}_{3} \mathrm{CN}\right)_{2}\right]^{+}$} \\
\hline & $\mathrm{m} / \mathrm{z}=154.14 \quad \mathrm{~m} / \mathrm{z}=\mathbf{4 2 9 . 1 0}$ \\
\hline & $\downarrow$ \\
\hline & {$\left[\mathrm{C}_{5} \mathrm{H}_{4} \mathrm{~N}_{4} \mathrm{~S}^{2+} \quad\left[\mathrm{MoOCl}_{2}\right]^{+}\right.$} \\
\hline & $\mathrm{m} / \mathrm{z}=77.51$ \\
\hline
\end{tabular}


[3]

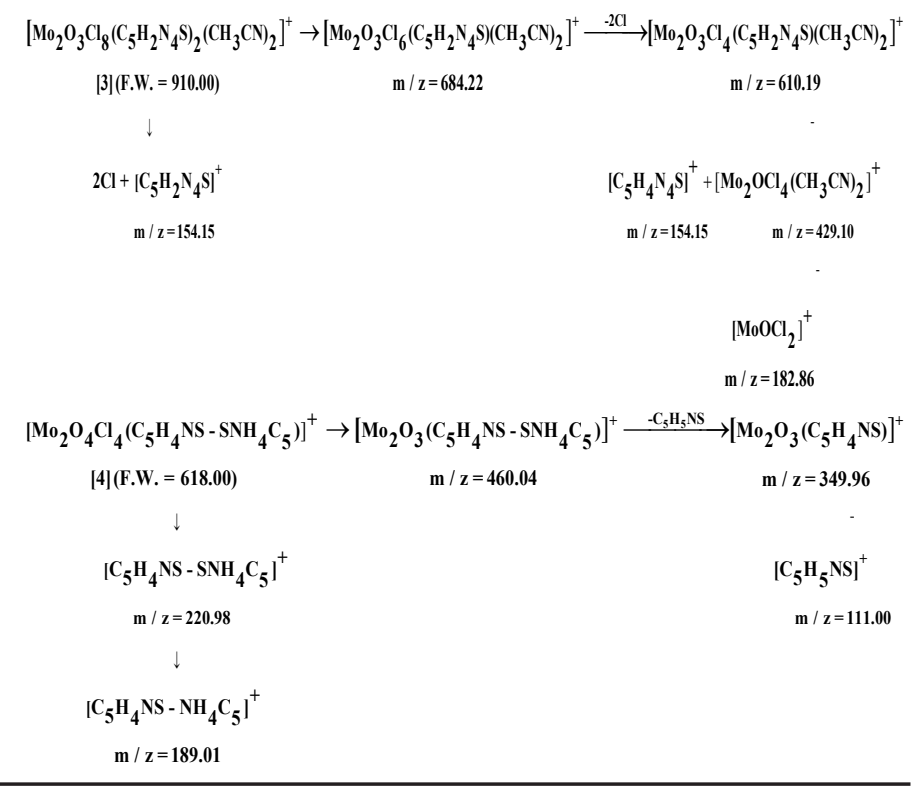

\section{Mass Spectra (LC-MS) ${ }^{59}$}

fragmentation ${ }^{60}$ of [4]. lons observed in mass spectrum There is formation of $\left[\mathrm{SOCl}_{2}\right]^{+}$and $[\mathrm{SOCl}]^{+}$on are concurrent with the depicted formulae (Tables 8, 9),

Table 9: ( $\mathrm{m} / \mathrm{z}$ values of some fragments)

\begin{tabular}{|c|c|c|c|c|}
\hline Comp. & Fragment & Calculated $^{52}$ & Observed & Relative abundance \\
\hline \multirow[t]{2}{*}{ [1] } & {$\left[\mathrm{SOCl}_{2}\right]^{+}$} & 117.9 & 118.9 & $100 \%$ \\
\hline & {$[\mathrm{SOCl}]^{+}$} & 82.93 & 84.0 & $17 \%$ \\
\hline \multirow[t]{6}{*}{ [2] } & {$\left[\mathrm{C}_{5} \mathrm{H}_{4} \mathrm{~N}_{4} \mathrm{~S}\right]^{+}$} & 152.01 & 154.14 & $100 \%$ \\
\hline & {$\left[\mathrm{C}_{5} \mathrm{H}_{4} \mathrm{~N}_{4} \mathrm{~S}^{2+}\right.$} & 76.00 & 77.51 & $26 \%$ \\
\hline & {$\left[\mathrm{MoOCl}_{2}\right]^{+}$} & 183.83 & 182.85 & $66 \%$ \\
\hline & {$\left[\mathrm{Mo}_{2} \mathrm{OCl}_{4}\left(\mathrm{CH}_{3} \mathrm{CN}\right)_{2}\right]^{+}$} & 433.73 & 429. & $3 \%$ \\
\hline & {$\left[\mathrm{Mo}_{2} \mathrm{O}_{3} \mathrm{Cl}_{4} \mathrm{C}_{5} \mathrm{H}_{2} \mathrm{~N}_{4} \mathrm{~S}\left(\mathrm{CH}_{3} \mathrm{CN}\right)_{2}\right]^{+}$} & 615.72 & 610.19 & $8 \%$ \\
\hline & {$\left[\mathrm{Mo}_{2} \mathrm{O}_{3} \mathrm{Cl}_{6} \mathrm{C}_{5} \mathrm{H}_{2} \mathrm{~N}_{4} \mathrm{~S}\left(\mathrm{CH}_{3} \mathrm{CN}\right)_{2}\right]^{+}$} & 685.66 & 684.21 & $4 \%$ \\
\hline \multirow[t]{6}{*}{ [3] } & {$\left[\mathrm{C}_{5} \mathrm{H}_{4} \mathrm{~N}_{4} \mathrm{~S}\right]^{+}$} & 152.01 & 154.15 & $100 \%$ \\
\hline & {$\left[\mathrm{MoOCl}_{2}\right]^{+}$} & 183.83 & 182.86 & $20 \%$ \\
\hline & {$\left[\mathrm{Mo}_{2} \mathrm{OCl}_{4}\left(\mathrm{CH}_{3} \mathrm{CN}\right)_{2}\right]^{+}$} & 433.73 & 437.21 & $5 \%$ \\
\hline & {$\left[\mathrm{Mo}_{2} \mathrm{O}_{3} \mathrm{Cl}_{4} \mathrm{C}_{5} \mathrm{H}_{2} \mathrm{~N}_{4} \mathrm{~S}\left(\mathrm{CH}_{3} \mathrm{CN}\right)_{2}\right]^{+}$} & 615.72 & 610.19 & $6 \%$ \\
\hline & {$\left[\mathrm{Mo}_{2} \mathrm{O}_{3} \mathrm{Cl}_{6} \mathrm{C}_{5} \mathrm{H}_{2} \mathrm{~N}_{4} \mathrm{~S}\left(\mathrm{CH}_{3} \mathrm{CN}\right)_{2}\right]^{+}$} & 685.66 & 684.22 & $3 \%$ \\
\hline & {$\left[\mathrm{Mo}_{2} \mathrm{O}_{3} \mathrm{Cl}_{8}\left(\mathrm{C}_{5} \mathrm{H}_{2} \mathrm{~N}_{4} \mathrm{~S}\right)_{2}\left(\mathrm{CH}_{3} \mathrm{CN}\right)_{2}\right]^{+}$} & -- & -- & -- \\
\hline \multirow[t]{5}{*}{ [4] } & {$\left[\mathrm{C}_{5} \mathrm{H}_{5} \mathrm{NS}\right]^{+}$} & 111.01 & 111.00 & $4 \%$ \\
\hline & {$\left[\mathrm{C}_{5} \mathrm{H}_{4} \mathrm{NS}-\mathrm{SN}_{4} \mathrm{C}_{5}\right]^{+}$} & 220.1 & 220.98 & $93 \%$ \\
\hline & {$\left[\mathrm{C}_{5} \mathrm{H}_{4} \mathrm{~N}-\mathrm{SN}_{4} \mathrm{C}_{5}\right]^{+}$} & 188.04 & 189.01 & $100 \%$ \\
\hline & {$\left[\mathrm{Mo}_{2} \mathrm{O}_{3}\left(\mathrm{C}_{5} \mathrm{H}_{4} \mathrm{NS}-\mathrm{SN}_{4} \mathrm{C}^{5}\right)\right]^{+}$} & 463.80 & 460.04 & $4 \%$ \\
\hline & {$\left[\mathrm{Mo}_{2} \mathrm{O}_{3}\left(\mathrm{C}_{5} \mathrm{H}_{4} \mathrm{NS}\right)\right]^{+}$} & 353.80 & 349.96 & $3 \%$ \\
\hline
\end{tabular}

\section{CONCLUSION}

[1] does not have any absorption in the region $2548-2602 \mathrm{~cm}^{-1}$ due to absence of $\mathrm{S}-\mathrm{H}$ group in it. $\mathrm{C}=\mathrm{S}$ stretching at 1261 and 1109 wave numbers are concurrent with presence of $\mathrm{C}=\mathrm{S}$ group. $761 \mathrm{~cm}^{-1}$ absorption due to C-S stretching support formation of Mo-S bonds. Terminal $\mathrm{v}(\mathrm{Mo}=\mathrm{O})$ at 986 $\mathrm{cm}^{-1}$ is observed. S-H peak has not been observed in
${ }^{1} \mathrm{H}$ NMR of [1]. This indicates that $\mathrm{S}-\mathrm{H}$ group is absent in [1]. Fragmentation pattern in GC-MS is compatible with the proposed formula.

[2] and [3] do not have any absorption around $2670 \mathrm{~cm}^{-1}$ because there is no S-H group in them. $\mathrm{S}-\mathrm{H}$ bond does not exist because of presence of $\mathrm{v}(\mathrm{C}=\mathrm{S})$ at $1027 \mathrm{~cm}^{-1}$ in both of them. lons observed in mass spectrum are concurrent with the depicted 
formulae. $\mathrm{S} \rightarrow$ Mo coordinate bond is likely to be present. Terminal $v(\mathrm{Mo}=\mathrm{O})$ absorbs at $973 \mathrm{~cm}^{-1}$ in [2] and at $970 \mathrm{~cm}^{-1}$ in [3]. S-H peak is missing in ${ }^{1} \mathrm{H}$ NMR of [2] and [3]. LC-MS supports the predicted formulae. $\mathrm{CH}_{3} \mathrm{CN}$ is present in in [2] and [3] as verified by the presence of its peak in ${ }^{1} \mathrm{H}$ NMR.

$\mathrm{N}-\mathrm{H}$ group absorbs at $3383 \mathrm{~cm}^{-1}$ in [4]. Absence of any peak around $2708 \mathrm{~cm}^{-1}$ is because of missing $\mathrm{S}-\mathrm{H}$ group it. $\mathrm{C}=\mathrm{S}$ stretching at $1261 \mathrm{~cm}^{-1}, 1109 \mathrm{~cm}^{-1}$ are due to $\mathrm{C}=\mathrm{S}$ group. C-S stretching at $767 \mathrm{~cm}^{-1}, 707 \mathrm{~cm}^{-1}$ depict Mo-S bond existence. $v(\mathrm{Mo}=\mathrm{O})$ absorption at $983 \mathrm{~cm}^{-1}$ in [4] suggests presence of terminal $\mathrm{Mo}=\mathrm{O}$ group it. Fragmentation pattern in LC-MS support the proposed formula.

\section{ACKNOWLEDGEMENT}

We thank Panjab University, Chandigarh (India) for providing testing facility.

\section{Conflict of interest}

The authors do not have any conflict of interest.

\section{REFERENCES}

1. https://medlineplus.gov/druginfo/meds/ a682653.html.

2. https://en.wikipedia.org/wiki/Mercaptopuri ne\#: : text=Mercaptopurine $\% 20(6 \% 2 D M P)$ $\% 2 \mathrm{C}$, Crohn's\%20disease $\% 2 \mathrm{C} \% 20$ and $\% 20$ ulcerative $\% 20$ colitis.

3. Łakomska, I.; Pazderski, L.; Sitkowsk, J.; Kozerski, L.; Pełczynska, M.; Nasulewicz, A.; Opolski, A.; Szłyk, E., J. Mol. Struct., 2004, 707, 241-247.

4. Blank, C.; Dabrowiak, J., J. Inorg. Biochem., 1984, 21, 21-29.

5. Cuin, A.; Massabni, A. C.; Pereira, G. A.; Leite, C. Q. F.; Pavan, F. R.; Sesti-Costa, R.; Heinrich, T. A.; Costa-Neto, C. M., Biomed. Pharmacother., 2011, 65, 334-338.

6. Bariyanga, J.; Luyt, A., J. Mol. Struct., 2001, 559, 49-54.

7. Cini, R.; Cinquantini, A.; Sabat, M.; Marzilli, L. G., Inorg. Chem., 1985, 24, 3903-3908.

8. Dubler, E.; Gyr, E., Inorg. Chem., 1988, 27, 1466-1473.

9. Abeer A. Sharfalddin,; Emwas, A. H.; Jaremko, M.; Hussien, M. A., Appl Organomet Chem., 2021, 35, 1-18, DOI: 10.1002/aoc.6041.

10. Anita M. Owczarzak, A. M.; Kubicki, M., Acta Crystallographica, Section E., 2012, E68, o1686. doi:10.1107/S1600536812020090.

11. Al-R. K. A.; Abdel-Aziz, H. A., Molecules., 2010, 15, 3775-3815.

12. Borhani, D. W.; Calderwood, D. J.; Frank, K. E. H.; Davis, M.; Josephsohn, N. S.; Skinner, B. S. WO Pat. 2008/063287, 2008.

13. Fidanze, S. D.; Erickson, S. A.; Wang, G. T.; Mantei, R.; Clark, R. F.; Sorensen, B. K.; Bamaung, N. Y.; Kovar, P.; Johnson, E. F.; Swinger, K. K.; Stewart, K. D.; Zhang, Q.; Tucker, L. A.; Pappano, W. N.; Wilsbacher, J. L.; Wang, J., Sheppard, G. S.; Bell, R. L.; Davidsen, S. K.; Hubbard, R. D., Bioorg. Med.
Chem. Lett., 2010, 20, 2452-2455.

14. Emmitte, K. A.; Wilson, B. J.; Baum, E. W.; Emerson, H. K.; Kuntz, K. W.; Nailor, K. E.; Salovich, J. M.; Smith, S. C.; Cheung, M.; Gerding, R. M.; Stevens, K. L.; Uehling, D. E.; Jr. Mook, R. A.; Moorthy, G. S.; Dickerson, S. H.; Hassell, A. M.; Leesnitzer, M. A.; Shewchuk, L. M.; Groy, A.; Rowand, J. L.; Anderson, K.; Atkins, C. L.; Yang, J.; Sabbatini, P.; Kumar, R. Bioorg. Med. Chem. Lett., 2009, 19, 1004-1007.

15. Andreani, A.; Burnelli, S.; Granaiola, M.; Guardigli, M.; Leoni, A.; Locatelli, A.; Morigi, R.; Rambaldi, M.; Rizzoli, M.; Varoli, L.; Roda, A. Eur. J. Med. Chem., 2008, 43, 657-661.

16. Andreani, A.; Rambaldi, M.; Mascellani, G.; Rugarli, P., Eur. J. Med. Chem., 1987, 22, 19-22.

17. Gupta, G. D., Jain, K. K., Gupta, R. P., Pujari, H. K., Indian J. Chem., Sect. B: Org. Chem. Incl. Med. Chem., 1983, 22, 268.

18. Amarouch, H., Loiseau, P. R., Bacha, C., Caujolle, R., Payard, M., Loiseau, P. M., Bories, C., Gayral, P., Eur. J. Med. Chem., 1987, 22, 463-466.

19. Andreani, A.; Rambaldi, M.; Andreani, F.; Bossa, R.; Galatulas, I., Eur. J. Med. Chem., 1988, 23, 385-389.

20. Andreani, A.; Rambaldi, M.; Locatelli, A.; Bossa, R.; Fraccari, A.; Galatulas, I., Pharm. Acta Helv., 1993, 68, 21-24.

21. Andreani, A.; Bonazzi, D.; Rambaldi, M., Arch. Pharm., 1982, 315, 451-456.

22. Andreani, A.; Rambaldi, M. M.; Locatelli, A.; Bossa, R.; Fraccari, A.; Galatulas, I., J. Med. Chem., 1992, 35, 4634-4637.

23. Ding, H.; Chen, Z.; Zhang, C.; Xin, T.; Wang, Y.; Song, H.; Jiang, Y.; Chen, Y. Xu, Y.; Tan, C. Molecules., 2012, 17, 4703-4716.

24. Abdelwareth Sarhan, A.O.; Al-Dhfyan, A.; Al-Mozaini, M. A.; Adra, C. N.; Aboul-Fadl, T. Eur. J. Med. Chem., 2010, 45, 2689-2694. 
25. Vu, C. B.; Bemis, J. E.; Disch, J. S.; Ng, P. Y.; Nunes, J. J.; Milne, J. C.; Carney, D. P.; Lynch, A. V.; Smith, J. J.; Lavu, S.; Lambert, P. D.; Gagne, D. J.; Jirousek, M. R.; Schenk, S.; Olefsky, J. M.; Perni, R. B., J. Med. Chem., 2009, 52, 1275-1283.

26. Poorrajab, F.; Ardestani, S. K.; Emami, S.; Behrouzi-Fardmoghaddam, M.; Shafiee, A.; Foroumadi, A. Eur. J. Med. Chem., 2009, 44, 1758-1762.

27. Khalaj, A., Nakhjiri, M., Negahbani, A. S., Samadizadeh, M., Firoozpour, L., Rajabalian, S., Samadi, N., Faramarzi, M. A., Adipour, N., Shafiee, A., Foroumadi, A., Eur. J. Med. Chem., 2011, 46, 65-70.

28. Kumaresan, K. L. Lu, S.; Wen, Y. S. ; Hwu, J. R., Organometallics., 1994, 13, 3170-3176.

29. Nagai, K.; Carter, B. J.; Xu, J.; Hecht, S. M., J. Am. Chem. Soc., 1991, 113, 5099-5100.

30. Lobana, T. S.; Bhatia, P. K., J. Sci. Ind. Res., 1989, 48, 394-401.

31. Singh, G.; Mangla, V.; Goyal, M.; Singla, K.; Rani, D., American International Journal of Research in Science, Technology, Engineering \& Mathematics., 2014, 8(2), 131-136.

32. Singh, G.; Mangla, V.; Goyal, M.; Singla, K.; Rani, D., American International Journal of Research in Science, Technology, Engineering \& Mathematics., 2015, 9(1), 25-33.

33. Singh, G.; Mangla, V.; Goyal, M.; Singla, K.; Rani, D., American International Journal of Research in Science, Technology, Engineering \& Mathematics., 2015, 10(4), 299-308.

34. Singh, G.; Mangla, V.; Goyal, M.; Singla, K.; Rani, D., International Congress on Chemical, Biological and Environmental Sciences., 2015, 930-942, May 7-9, Kyoto (Japan).

35. Singh, G.; Mangla, V.; Goyal, M.; Singla, K.; Rani, D.; Kumar, R., American International Journal of Research in Science, Technology, Engineering \& Mathematics., 2016, 16(1), 56-64.

36. Singh, G.; Kumar, R., American International Journal of Research in Science, Technology, Engineering \& Mathematics., 2018, 22(1), 01-08.

37. Rani, D.; Singh, G.; Sharma, S., Oriental Journal of Chemistry., 2020, 36(6), 1096-1102.

38. Mangla, V.; Singh, G., American International Journal of Research in Science, Technology, Engineering \& Mathematics., 2019, 26(1), 145-148.

39. Mangla, V.; Singh, G., Oriental Journal of Chemistry., 2019, 35(3), 1094-1102.

40. Vogel, A. I., A text book of Quantitative Inorganic Analysis; John Wiley and Sons:
New York, 1963(Standard methods).

41. Jolley, J.; Cross, W. I.; Pritchard, R. G.; McAuliffe, C.A.; Nolan, K. B., Inorganica Chimica Acta., 2001, 315, 36-43.

42. Kahn, E. S.; Rheingold, A. L.; Shupack, S. I., J. Crystallographic and Spectroscopic Research., 1993, 23(9), 697-710.

43. Trzhtsinskaya, B. V.; Abramova, N. D., J. Sulphur Chemistry., 1991, 10(4), 389-430.

44. Liang Ying-Qiu; Zhao Wen-Yun; XU Wei-Qing, Acta Chimica Sinica., 1986, 1126, 42-47.

45. Barraclough, C. G.; Kew, D. J., Australian J. Chem., 1970, 23, 2387-2396.

46. Ward, B. G.; Stafford, F. E., Inorg. Chem., 1968, 7, 2569.

47. Bodo, H. H.; Regina, Z. Chem., 1976, 16, 407.

48. Abramenko, V. L.; Sergienko, V. S., Russian J. Inorg. Chem., 2009, 54(13), 2031-2053.

49. Ueyama, N.; Nakata, M.; Araki, T.; Nakamura, A., Chemical Soc. Japan, Chemistry Lett., 1979, 421-424.

50. Kumar, G. P.; Sanganal, J. S.; Phani, A. R.; Tripathi, S. M.; Manohara, C.; Raghavendra, H. L.; Janardhana, P. B.; Amaresha, S.; Swamy, K. B.; Prasad, R. G. S. V., Phamacological Research., 2015, 100, 47-57.

51. http://www.sigmaaldrich.com/catalog/product/ aldrich/m5852?lang=en\&region=IN.

52. Refat, M. S.; Farias, R. F. D., J. Serb. Chem. Soc., 2006, 71(12), 1289-1300.

53. Hanif, M.; Saddiq, A.; Hasnain, S.; Ahmad, S.; Rabbani, G.; Isab, A. A., Spectroscopy., 2008, 22, 51-56.

54. Xhang, H. L.; Evans, S. D.; Henderson, J. R.; Miles, R. E.; Shen, T., J. Phys. Chem. B., 2003, 107, 6087-6095.

55. Shpakovsky, D. B.; Banti, C. N.; Houle, G. B.; Kourkoumelis, N.; Manoli, M.; Manos, M. J.; Tasiopoulos, A. J.; Hadjikakou, S. K.; Milaeva, E. R.; Charalabopoulos, K.; Bakas, T.; Butlerd, I. S.; Hadjiliadisa, N., Dalton Trans., 2012, 41, 14568-14582.

56. Abramenko, V. L.; Sergienko, V. S.; Churakov, A. V., Russian J. Coord. Chem., 2000, 26(12), 866-871.

57. Sharma, M.; Koty, A.; Srivastava, M.; Srivastava, A., J. Chinese Chemical Society., 2007, 54, 1419-1432.

58. http://www.molbase.com/en/hnmr_6857-347-moldata-838140.html\#tabs.

59. http://www.sisweb.com/referenc/tools/ exactmass.htm.

60. https://webbook.nist.gov/cgi/cbook. cgi?ID=C7719097\&Mask=28F. 